\title{
Tracking Control of a Galfenol-Actuated Nanopositioning Stage Using Feedforward Control with a Disturbance Observer
}

\author{
Shiping Jiang, ${ }^{1}$ Bin Xu $\mathbb{D}{ }^{2}$ Shuxin Liu, ${ }^{3}$ and Wei Zhu ${ }^{4}{ }^{4}$ \\ ${ }^{1}$ Faculty of Civil Engineering and Mechanics, Jiangsu University, Zhenjiang, Jiangsu, 212013, China \\ ${ }^{2}$ School of Manufacturing Science and Engineering, Sichuan University, 610065 Chengdu, China \\ ${ }^{3}$ The South Western Technical Institute of Physics, Chengdu 610041, China \\ ${ }^{4}$ Institute of Launch Dynamics, Nanjing University of Science and Technology, Nanjing 210094, China \\ Correspondence should be addressed to Bin Xu; xubinchuangda@163.com
}

Received 20 June 2019; Accepted 21 October 2019; Published 26 November 2019

Academic Editor: Zhonghua Yao

Copyright ( $\odot 2019$ Shiping Jiang et al. This is an open access article distributed under the Creative Commons Attribution License, which permits unrestricted use, distribution, and reproduction in any medium, provided the original work is properly cited.

\begin{abstract}
The main challenge of the galfenol actuator for high-precision positioning is the inherent nonsmooth hysteresis, which may lead to undesirable inaccuracies or oscillations and even instability. The primary aim of this study is to develop a tracking control method to precisely control the output displacement of a galfenol-actuated nanopositioning stage using feedforward control with a disturbance observer. In order to accurately describe the rate-dependent hysteresis, considering the dynamic behavior of the power amplifier, a novel dynamic model is put forward. Then, a developed controller is designed. In this controller, a feedforward control is developed to compensate the rate-dependent hysteresis, and a disturbance observer is employed to restrain disturbances, high-order unmodeled dynamics, and hysteresis compensation error. The comparative experimental results show that the proposed control method can significantly improve the positioning accuracy and suppress disturbances. This research can be applied in various micro and nanopositioning and vibration control fields.
\end{abstract}

\section{Introduction}

Magnetostrictive materials have received much attention in recent years due to their advantages of fast response, large displacement output, and the fact that they are well-suited for actuation purposes. Galfenol alloy is a structural magnetostrictive material which is suitable for load carrying transducers operating under combined loads $[1,2]$, and it has been applied to develop nanopositioning actuators for industrial $[3,4]$, aerospace $[5,6]$, and manufacturing applications [7].

Unfortunately, the main challenge of the galfenol actuator for high-precision positioning is the inherent nonsmooth hysteresis, which may lead to undesirable inaccuracies or oscillations and even instability $[8,9]$. The feedforward control based on the hysteresis model is an effective way to address the hysteresis nonlinearity problem for a galfenol actuator, especially when the system is operating in highly hysteresis. The main idea of the feedforward control is to construct an inverse hysteresis model to compensate the hysteresis, and then the traditional linear control methods can be applied to control the linear dynamic system $[10,11]$. The hysteresis model includes the well-known Preisach model [12], Prandtl-Ishlinskii model [13], and Bouc-Wen model [14].

Such hysteresis models are not able to capture the timevarying effect of the command signal on the dynamic force response of the galfenol-actuated stage due to the rate-dependent hysteresis of the galfenol actuator that suggests that the hysteresis strongly depends on the varying rate of the command signal applied on the power amplifier of the galfenol actuator. To model this rate-dependent effect, numerous improvement strategies based on these standard hysteresis models are proposed by researchers [15]. However, since the dynamic characteristics of the amplifier are not considered, it is still extremely difficult for these models to provide satisfactory accuracy over a wide frequency range.

In addition, as a matter of fact, it is generally impossible to obtain the perfect hysteresis and dynamic model due to 
the existence of the modeling uncertainties and disturbances. The dynamics of the galfenol-actuated stage with the hysteresis compensation can be treated as a linear dynamic system plus a lumped disturbance term.

The primary aim of this study is to develop a tracking control method to precisely control the output displacement of a galfenol-actuated nanopositioning stage using feedforward control with a disturbance observer. In order to accurately describe the rate-dependent hysteresis, a novel dynamic model is put forward by considering the dynamic behavior of the power amplifier. Then, a feedforward control using a hysteresis observer (HOB) is developed to compensate the rate-dependent hysteresis. Finally, a disturbance observer (DOB) is employed to restrain disturbance.

This paper is organized as follows. In Section 2, the dynamic model of the galfenol-actuated nanopositioning stage is presented. Then, in the next section the developed controllers are designed. After that, Section 4 provides the comparative experimental results. Finally, Section 5 concludes this paper.

\section{Electromechanical Coupling Model of a Galfenol-Actuated Nanopositioning Stage}

Figure 1 shows a practical galfenol-actuated nanopositioning stage. Both ends of the galfenol alloy beam are fixed to the base by the fixed pieces. There are a set of coils on the base to drive the stage. The load is bonded with the beam in the middle. When applying a driving current to the stage, the magnetostrictive force acting on the beam due to the magnetostriction effect leads to a forced vibration of the load.

Schematically, the electromechanical coupling model of the galfenol-actuated stage is illustrated in Figure 2. This model contains three components to fully represent the dynamic behavior of the stage: an electric model to represent the dynamic behavior of the power amplifier; a hysteresis model to describe the magnetostriction effect; and a mechanical model to capture the dynamic characteristics of the stage.

2.1. Electric Model of the Power Amplifier. In general, the galfenol actuator can be equivalent to be an inductance in tandem with a resistance in the electric circuit. Considering the dynamics of the power amplifier, the relationship between the command voltage $u_{\mathrm{c}}(t)$ and practical driving current $I_{\mathrm{g}}(t)$ applied on the galfenol actuator can be expressed by

$$
\left(R_{\mathrm{g}}+R_{\mathrm{p}}\right) I_{\mathrm{g}}(t)+L_{\mathrm{g}} \frac{\mathrm{d} I_{\mathrm{g}}(t)}{\mathrm{d} t}=K_{\mathrm{p}} u_{\mathrm{c}}(t)
$$

where $R_{\mathrm{p}}$ and $K_{\mathrm{p}}$ are the equivalent resistance and amplification gain of the power amplifier and $L_{\mathrm{p}}$ and $R_{\mathrm{p}}$ are the inductance capacitance and resistance of the galfenol actuator. Taking Laplace transformation equation (1), the relationship between the command voltage and driving current can be expressed in the $s$-domain by

$$
G_{\mathrm{p}}(s)=\frac{I_{\mathrm{g}}(s)}{U_{\mathrm{c}}(s)}=\frac{K_{1}}{1+\tau s},
$$

where $K_{1}=K_{\mathrm{p}} / R_{\mathrm{g}}+R_{\mathrm{p}}$ and $\tau=L_{\mathrm{g}} / R_{\mathrm{g}}+R_{\mathrm{p}}$.



FIGURE 1: Galfenol-actuated nanopositioning stage.

2.2. Mechanical Model of the Stage. With respect to the mechanical dynamic of the stage, it can be equivalent to be a second-order system described by

$$
m \ddot{x}(t)+c \dot{x}(t)+k x(t)=F_{\mathrm{g}}(t),
$$

where $m$ is the equivalent moving mass, $c$ is the damping coefficient, $k$ is the stiffness, and $F_{\mathrm{g}}$ is the driving force applied by the galfenol actuator. Taking Laplace transformation of the relationship in equation (3), the nominal transfer function of the mechanical system can be obtained in the $s$-domain as

$$
G_{s}(s)=\frac{X(s)}{F_{\mathrm{g}}(s)}=\frac{k_{2} \omega^{2}}{s^{2}+2 \xi \omega s+\omega^{2}},
$$

where $k_{2}=1 / k, \omega=\sqrt{k / m}$, and $\xi=c / 2 \sqrt{m k}$.

Obviously, this equivalent ignores the high-order part of the dynamics, which is treated as an unknown disturbance and compensated by a DOB (see Section 3.2).

2.3. Hysteresis Model of the Galfenol Actuator. Due to the magnetostriction effect, a combination of linear force and hysteresis force will be generated by the galfenol actuator under a driving current. The Bouc-Wen model is proposed to represent the magnetostriction effect and can be given by

$$
\begin{aligned}
& F_{\mathrm{g}}=F_{1}+F_{\mathrm{h}}=K_{\mathrm{l}} i_{\mathrm{g}}+K_{\mathrm{h}} h, \\
& \dot{h}=\left\{\alpha-\left[\beta s g n\left(\dot{I}_{\mathrm{g}} h\right)+\gamma\right]|\dot{h}|^{n}\right\} \dot{I}_{\mathrm{g}},
\end{aligned}
$$

where $F_{\mathrm{g}}$ is the magnetostriction force of the galfenol actuator; $F_{1}$ and $F_{\mathrm{h}}$ are the linear force and hysteresis force, respectively; $K_{1}$ is a constant ratio between the linear force and the driving current; $h$ is the hysteresis current; $K_{\mathrm{h}}$ is a constant ratio between the hysteresis force and the hysteresis current; $\alpha, \beta, \gamma$, and $n$ are the parameters of the Bouc-Wen hysteresis operator; and $\operatorname{sgn}(x)=\left\{\begin{array}{l}1, x>0 \\ -1, x<0\end{array}\right.$.

\section{Controller Design}

In this section, the control method which consists of a hysteresis compensator and a disturbance compensator is discussed in detail.

3.1. Hysteresis Compensation Using a HOB. We focus on the feedforward compensation of the hysteresis using a HOB in 




FIGURE 2: Electromechanical coupling model of the stage.

this section. The desired tracking signal $x_{\mathrm{d}}(t)$ is applied to the hysteresis compensator which consists of the inverse mechanical model $G_{s}^{-1}(s)$, the $\mathrm{HOB}$, and the inverse electric model $G_{\mathrm{p}}^{-1}(s)$. On the basis of the analysis in Section 2, since the hysteresis model is used to describe the relationship between the output force and the driving current, we employ the Bouc-Wen model to observe and compensate the hysteresis force. This process can be written as follows:

$$
\begin{aligned}
& F_{\mathrm{c}}=F_{\mathrm{g}}-\widehat{F}_{\mathrm{h}}=F_{1}+K_{\mathrm{h}}(h-\widehat{h})=K_{\mathrm{l}} I_{\mathrm{g}}+e_{\mathrm{c}}, \\
& \dot{\hat{h}}=\left\{\alpha-\left[\beta \operatorname{sgn}\left(\dot{I}_{\mathrm{g}} \widehat{h}\right)+\gamma\right]|\widehat{h}|^{n}\right\} \dot{I}_{\mathrm{g}},
\end{aligned}
$$

where $\widehat{F}_{\mathrm{h}}$ is the estimated value of the hysteresis force and $e_{\mathrm{c}}$ is the compensation error.

Equation (6) can be rewritten as

$$
\widehat{I}_{\mathrm{g}}=\frac{F_{\mathrm{c}}}{K_{l}}
$$
by

Then, the command signal of the controller can be given

$$
\left\{\begin{array}{l}
u_{\mathrm{c}}=G_{\mathrm{p}}^{-1} \widehat{I}_{\mathrm{g}}=\frac{G_{\mathrm{p}}^{-1}}{K_{l}}\left(G_{s}^{-1} x_{\mathrm{d}}-K_{\mathrm{h}} \widehat{h}\right), \\
\dot{\widehat{h}}=\left\{\alpha-\left[\beta \operatorname{sgn}\left(\dot{I}_{\mathrm{g}} \widehat{h}\right)+\gamma\right]|\widehat{h}|^{n}\right\} \dot{I}_{\mathrm{g}} .
\end{array}\right.
$$

The compensated system with the $\mathrm{HOB}$ can be approximated as a linear dynamics model $G_{s}(\mathrm{~s})$, as shown in Figure 3. In practice, it is generally impossible to obtain the perfect linear dynamics model due to the existence of the modeling errors and disturbances. Therefore, in the next section, the disturbance compensation control using a DOB is designed.

3.2. Disturbance Compensation Using a DOB. We treat the external disturbance, high-order unmodeled dynamics, and compensation error of the hysteresis as unknown disturbances added to a linear system. A DOB is designed to realtime observe this unknown disturbances.

The scheme framework of the disturbance compensation using a DOB depicted in Figure 4. As shown in Figure 4, the estimated value of the disturbance can be expressed by

$$
\widehat{d}=\frac{Q G_{s}^{-1}\left(x_{d}-x\right)}{1-Q},
$$

where $Q$ is a low-pass filter. It should be pointed out that the DOB can estimate disturbances precisely if these disturbances stay within the bandwidth of the low-pass $Q$ filter [16]. In order to precisely observe the disturbances, it is desired that $Q(j \omega) \approx 1$ in an extensive frequency range. Unfortunately, the bandwidth of the $Q$ filter is limited by the robustness of the system [17].

Therefore, considering the relative degree of the mechanical model $G_{s}$ and the robustness of the system, a two order low-pass filter is adopted, which can be expressed by

$$
Q(s)=\frac{1}{\left(\left(1 / \omega_{Q}\right) s\right)^{2}+2\left(1 / \omega_{Q}\right) s+1}
$$

where $\omega_{Q}$ is the cutoff frequency of the low-pass filter. It means the lumped disturbance and model uncertainties whose frequencies are lower than the cutoff frequency $\omega_{Q}$ can be approximately restrained.

In summary, the control strategy combined with the hysteresis and disturbance compensators can be expressed as

$$
\left\{\begin{array}{l}
u_{\mathrm{c}}=\frac{G_{\mathrm{p}}^{-1}}{K_{\mathrm{l}}}\left(G_{s}^{-1} x_{\mathrm{d}}-K_{\mathrm{h}} \widehat{F}_{\mathrm{h}}+\frac{\mathrm{Q} G_{s}^{-1}\left(x_{\mathrm{d}}-x\right)}{1-\mathrm{Q}}\right), \\
\dot{\widehat{F}}_{\mathrm{h}}=\left\{\alpha-\left[\beta \operatorname{sgn}\left(\dot{I}_{\mathrm{g}} \widehat{F}_{\mathrm{h}}\right)+\gamma\right]\left|\widehat{F}_{\mathrm{h}}\right|^{n}\right\} \dot{I}_{\mathrm{g}} .
\end{array}\right.
$$

Due to the controller consisting of two compensators, the stability of the system can be guaranteed by selecting the $Q$ filter with a suitable bandwidth.

\section{Experimental Verification}

4.1. Experimental Setup. In order to experimentally validate the proposed DOB controller with the HOB, the experimental setup is shown in Figure 5. It can be seen from Figure 5, the experimental setup is composed of the galfenolactuated nanopositioning stage, power amplifier, function generator (type: 33220A, Agilent corporation), real-time control system (dSPACE DS1006 with MATLAB/Simulink), and laser Doppler vibrometer (LDV, type: OFV-505/OFV- 


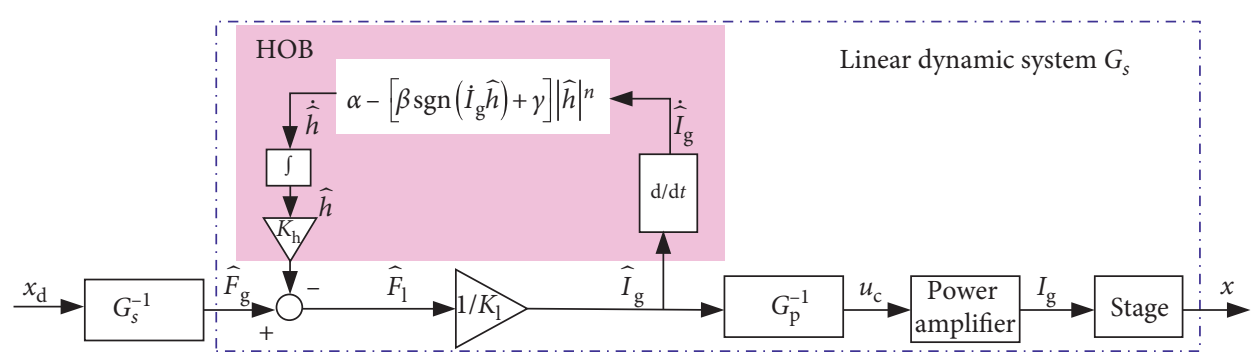

Figure 3: Scheme framework of the hysteresis compensation.

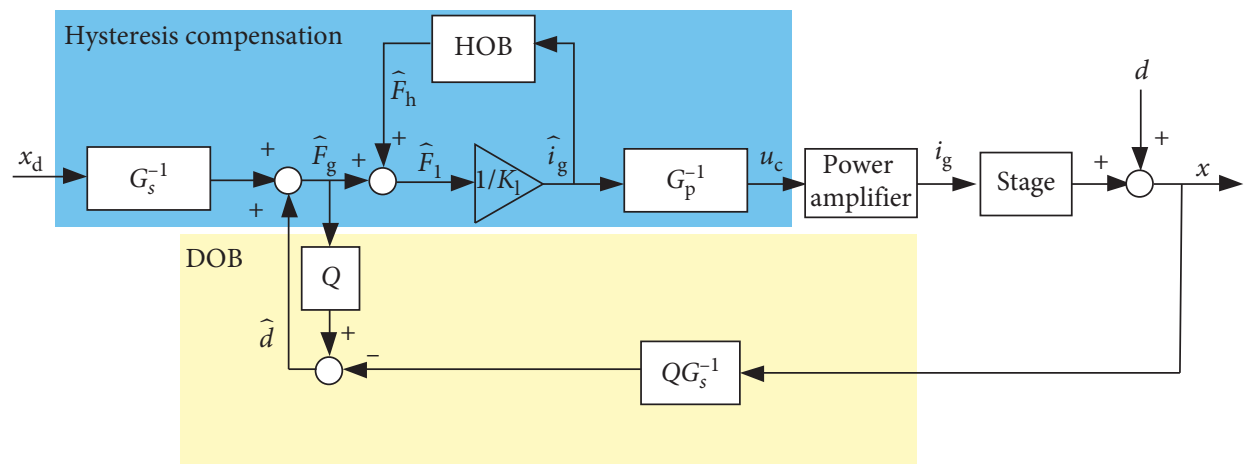

FIGURE 4: Scheme framework of the disturbance compensation.

5000, Polytec corporation). The external disturbances are generated by the function generator.

4.2. Modeling Results. Through sweep excitation, the amplitude-frequency and phase-frequency functions are obtained for the dynamics model identification. Through identifying the resonant frequency, resonant peak, and phase change, the dynamic parameters of the stage and power amplifier are obtained:

$$
\begin{aligned}
G_{\mathrm{s}}(s) & =\frac{32.95}{s^{2}+866 s+9.57 \times 10^{4}}, \\
G_{\mathrm{p}}(s) & =\frac{0.2}{0.0015 s+1} .
\end{aligned}
$$

With the hysteresis model, using the reported identification method in [18], the best-fit parameters of the Bouc-Wen model are summarized in Table 1.

In order to examine the rate-dependent characteristic and the modeling accuracy of the proposed electromechanical coupling model, harmonic command voltages with amplitude of $10 \mathrm{~V}$ and frequencies of 1 and $100 \mathrm{~Hz}$ are employed. The practical and estimated responses from both the electromechanical model and traditional model, which does not take into account the dynamic characteristics of the power amplifier, are illustrated in Figure 6.

From the results shown in Figure 6, the modeling accuracy for the two models with driving frequency of $1 \mathrm{~Hz}$ is similar. The modeling errors with driving frequency of $1 \mathrm{~Hz}$ reach to the largest which are $0.068 \mu \mathrm{m}$ and $0.876 \mu \mathrm{m}$ for the electromechanical and traditional models, respectively. Although only static hysteresis models are employed by the electromechanical model, a good consistence between the practical and estimated results is observed. Good modeling accuracy using the electromechanical model suggests that this model can predict the rate-dependent behavior of the stage well up to $100 \mathrm{~Hz}$. However, the traditional model does not possess this capacity. Figure 7 shows the Bode diagram of the model and stage, which also indicates that the model can predict the rate-dependent behavior of the stage well up to $100 \mathrm{~Hz}$. The model cannot describe the dynamic performance of the stage under frequency higher than $100 \mathrm{~Hz}$, and the modeling error will become larger.

\subsection{Control Performance}

4.3.1. Feedforward Control Results. The effectiveness of the feedforward hysteresis compensator is investigated in this section. Because the highest frequency of the stage in this study is $100 \mathrm{~Hz}$, we select that the cutoff frequency $\omega_{Q}$ of the $Q$ filter is $500 \mathrm{~Hz}$. The variations in measured stage displacement with the input current applied to the feedforward control are illustrated in Figure 8 for different excitation frequencies. Figure 8 shows that the proposed HOB control can effectively compensate for the hysteresis of the stage under different input frequencies.

4.3.2. Without External Disturbances. In this case, the DOB is mainly used to compensate for high-order unmodeled dynamics and hysteresis compensation error. For harmonic tracking target with different frequencies, the control resulted using the $\mathrm{HOB}$ controller and $\mathrm{HOB}+\mathrm{DOB}$ controller are illustrated in Figure 9. It can be observed in Figure 9, both the $\mathrm{HOB}$ controller and $\mathrm{HOB}+\mathrm{DOB}$ controller can obtain the high positioning 


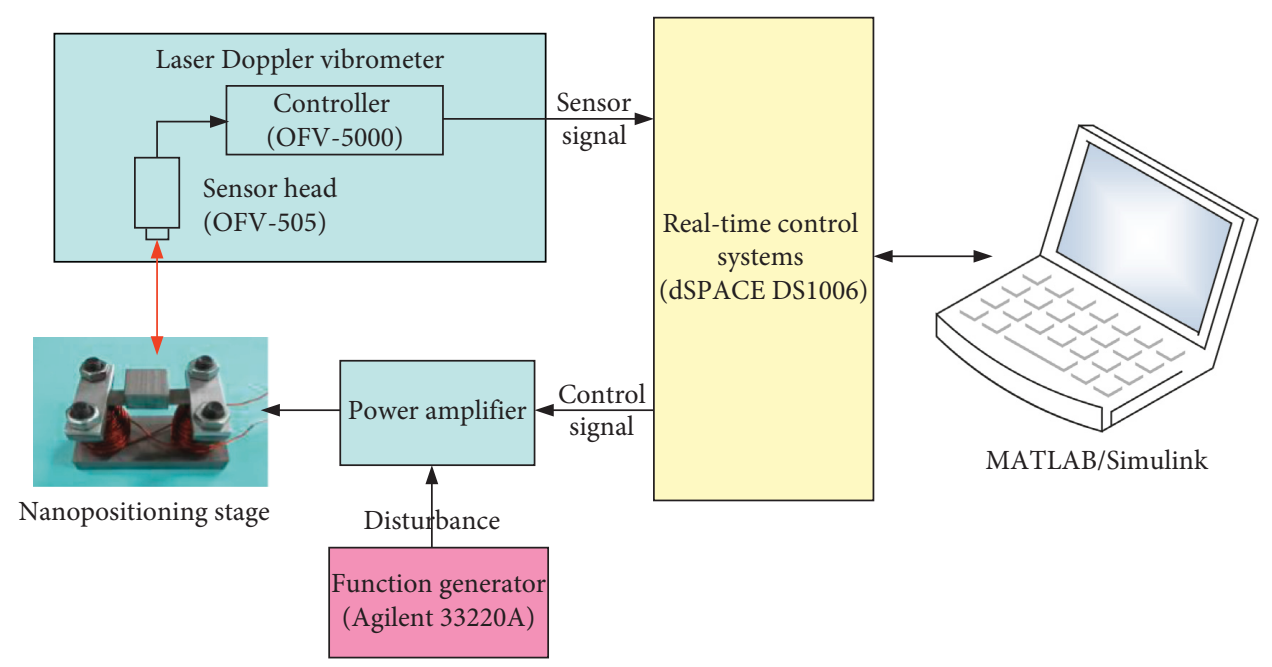

(a)

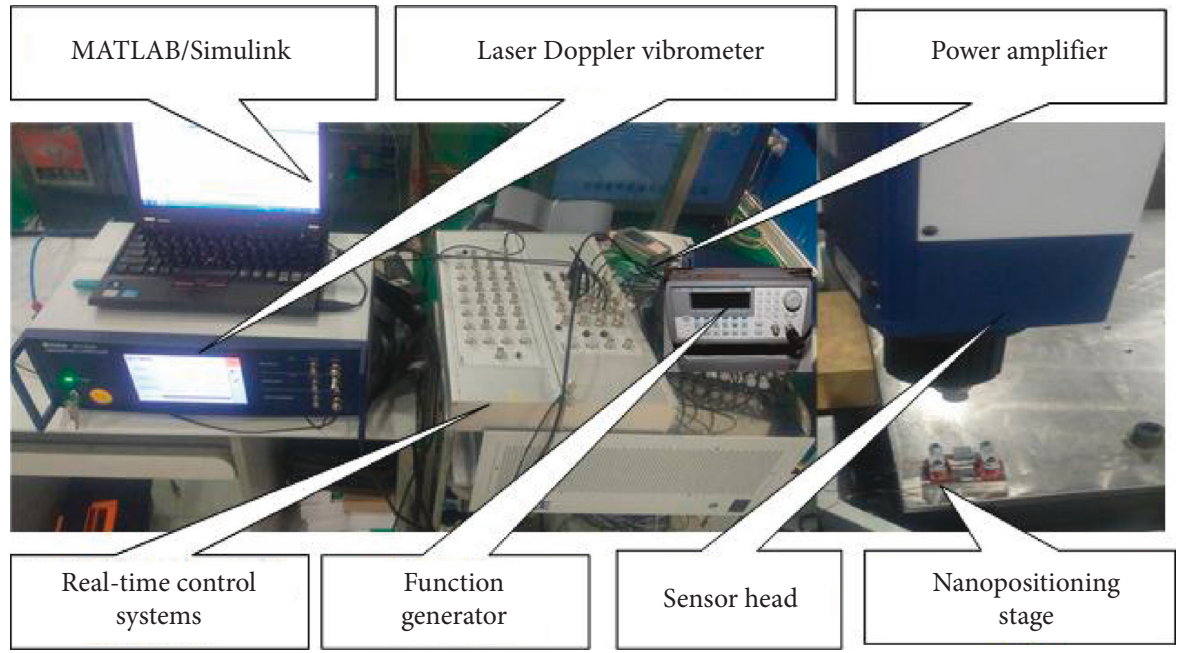

(b)

Figure 5: Rapid control prototypes of the proposed control method. (a) Schematic. (b) Photograph.

TABLE 1: Identified parameters of the Bouc-Wen model.

\begin{tabular}{lccccc}
\hline$K_{1}$ & $K_{\mathrm{h}}$ & $\alpha$ & $\beta$ & $\gamma$ & $n$ \\
\hline 0.0069 & 0.0085 & -0.45 & 0.31 & -0.11 & 1.12 \\
\hline
\end{tabular}

accuracy. The maximum absolute control errors are summarized in Table 2. The maximum control error of the $\mathrm{HOB}$ controller with driving frequency of $100 \mathrm{~Hz}$ increases from $0.082 \mu \mathrm{m}$ to $0.125 \mu \mathrm{m}$. The reason for this phenomenon is that the modeling error caused by high-frequency unmodeled dynamics increases as the drive frequency increases. The maximum control error of the $\mathrm{HOB}+\mathrm{DOB}$ controller is $0.056 \mu \mathrm{m}$. Obviously, the DOB can compensate for high-order unmodeled dynamics and hysteresis compensation error.

Furthermore, a complex tracking target with multiple harmonic signals with different amplitudes and frequencies is also employed to verify the effectiveness of the control method. The control results are shown in Figure 10. The maximum absolute control errors are also summarized in
Table 2. The maximum absolute control error for the HOB and $\mathrm{HOB}+\mathrm{DOB}$ controllers are $0.131 \mu \mathrm{m}$ and $0.052 \mu \mathrm{m}$, respectively. With respect to the complex signal, the control error resulted from the $\mathrm{HOB}+\mathrm{DOB}$ controller is similar to that of tracking simple harmonic signals, suggesting that the $\mathrm{HOB}+\mathrm{DOB}$ controller is powerful for controlling the galfenol-actuated nanopositioning stage.

4.3.3. With External Disturbances. In order to verify the disturbance suppression ability of the DOB, an external disturbance generated by the function generator is employed to disturb the complex tracking target. The control results are shown in Figure 11, and the maximum absolute control errors are also summarized in Table 2. The maximum 


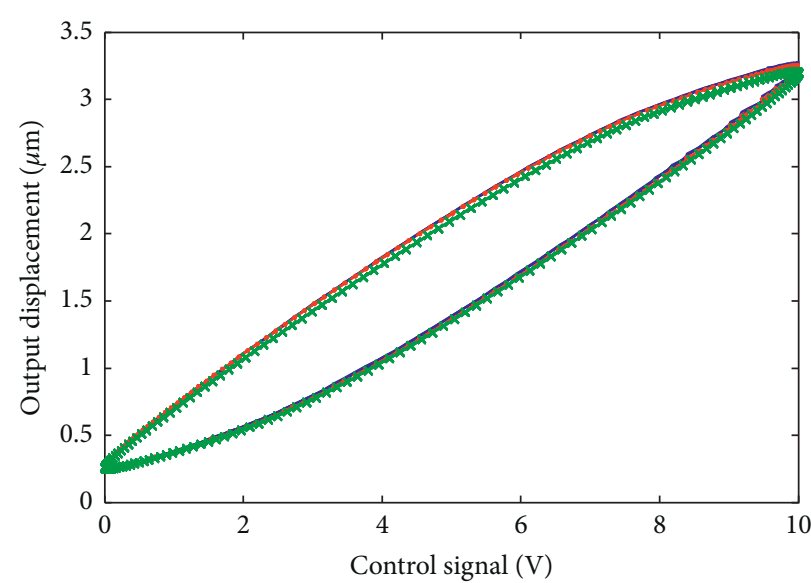

- Experimental $(1 \mathrm{~Hz})$

-.-. Electromechanical model $(1 \mathrm{~Hz})$

* Traditional model $(1 \mathrm{~Hz})$

(a)

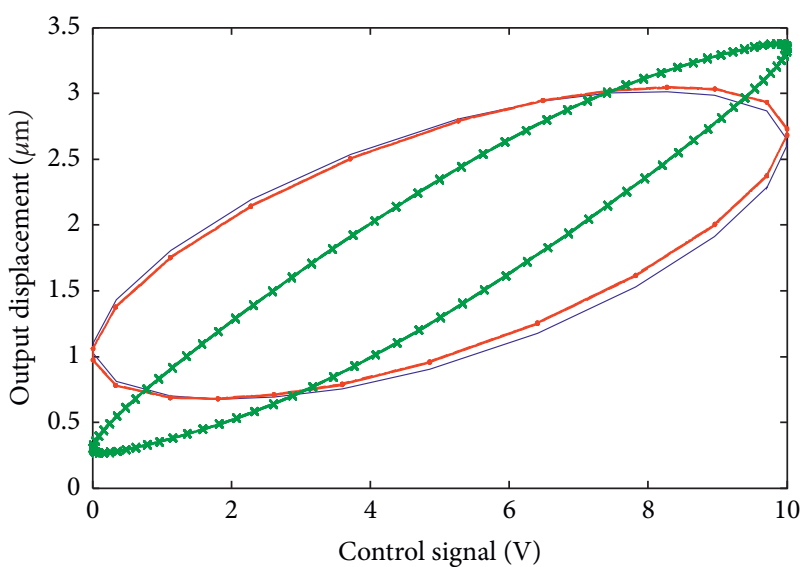

- Experimental $(100 \mathrm{~Hz})$

-..- Electromechanical model $(100 \mathrm{~Hz})$

* Traditional model $(100 \mathrm{~Hz})$

(b)

FIGURE 6: Comparisons of hysteresis characteristics between the experimental and the modeling results under sinusoidal control signals with different frequencies. (a) $1 \mathrm{~Hz}$. (b) $100 \mathrm{~Hz}$.

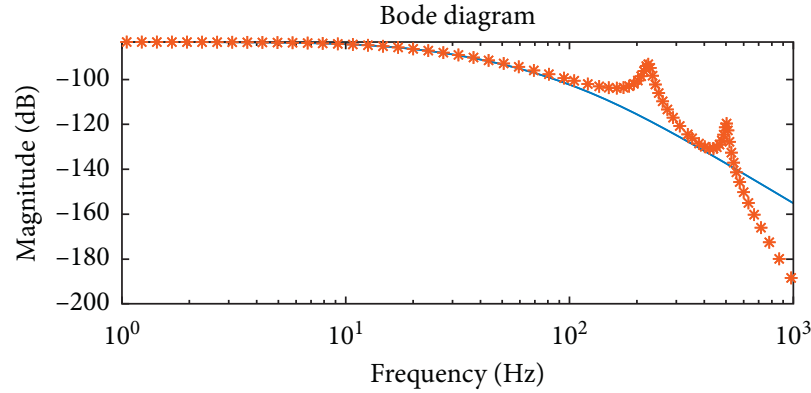

- Model

* * Experimental

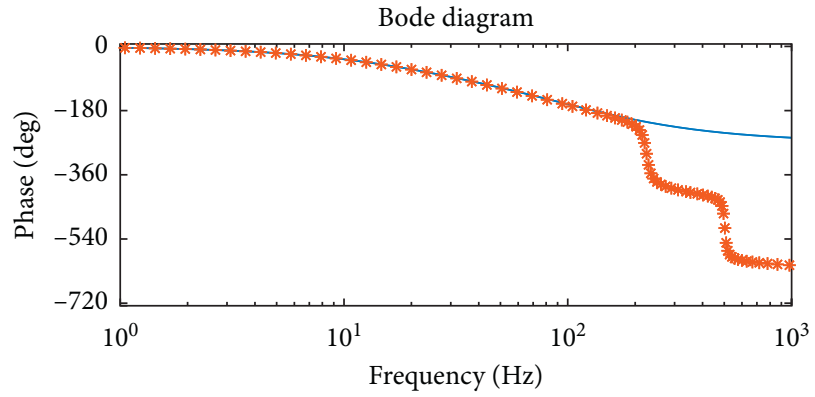

- Model

* * Experimental

(a)

(b)

Figure 7: Amplitude-frequency and phase-frequency characteristics.

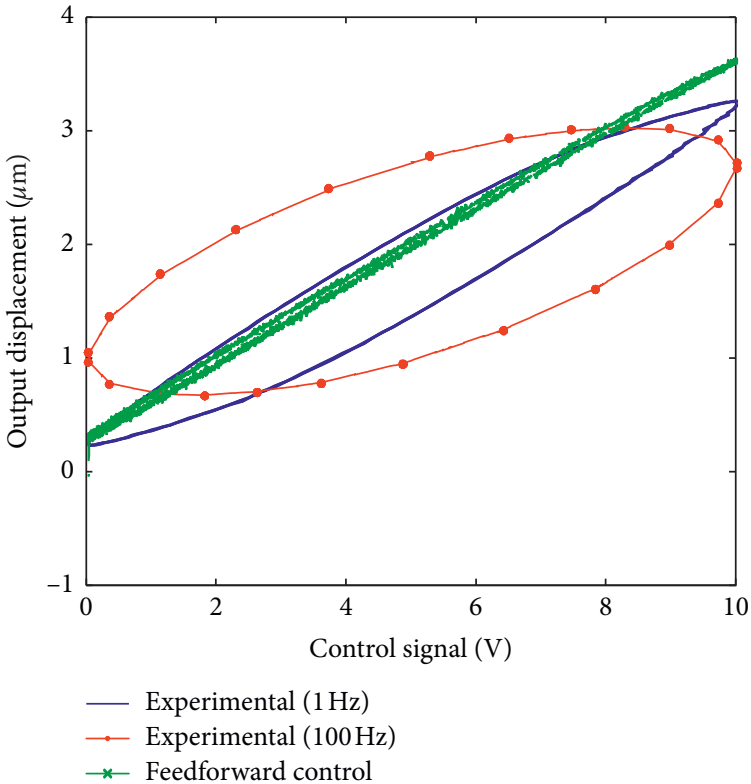

FIgURE 8: Comparisons of the output-input characteristics of the stage with and without the feedforward control. 


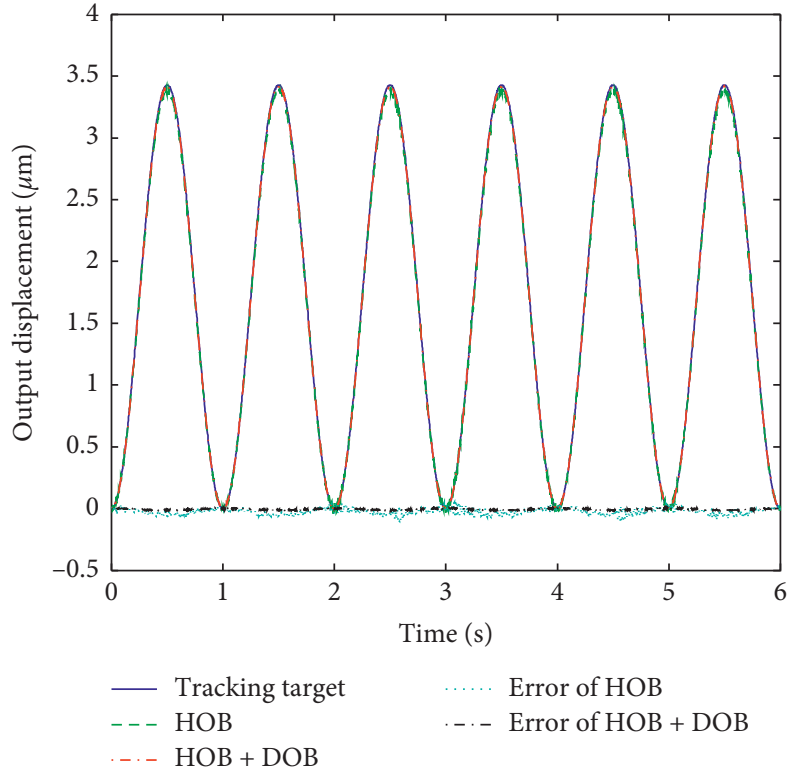

(a)

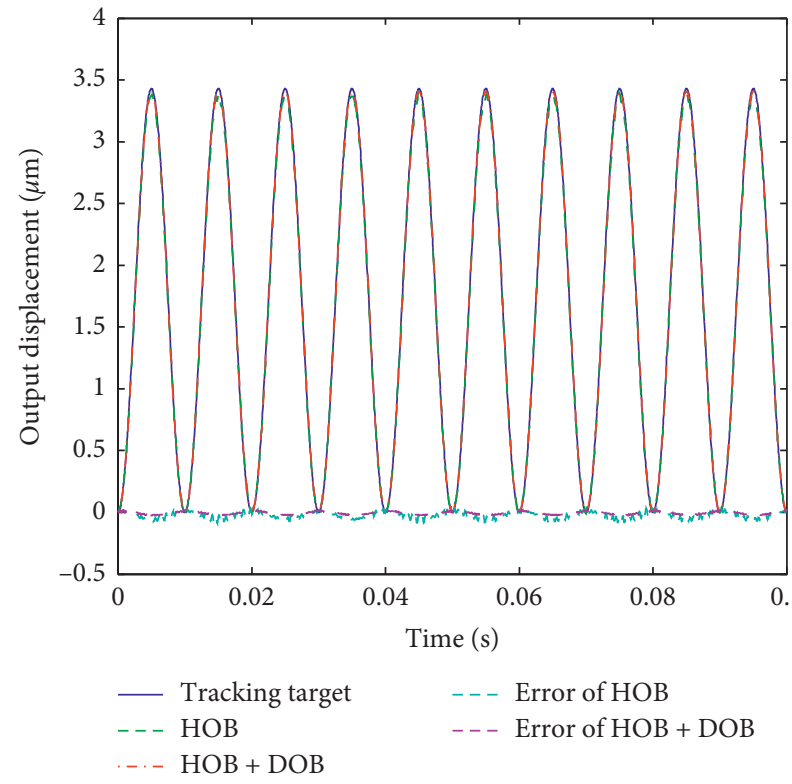

(b)

Figure 9: Control results in tracking sinusoidal targets. (a) $1 \mathrm{~Hz}$. (b) $100 \mathrm{~Hz}$.

TABle 2: Maximum absolute control errors of the proposed control methods.

\begin{tabular}{lrr}
\hline Tracking target & & Maximum absolute control errors $(\mu \mathrm{m})$ \\
& HOB & 0.049 \\
$1 \mathrm{~Hz}$ & 0.082 & 0.056 \\
$100 \mathrm{~Hz}$ & 0.125 & 0.052 \\
Nonperiodic & 0.131 & 0.059 \\
Nonperiodic + disturbance & 0.259 & DOB \\
\hline
\end{tabular}

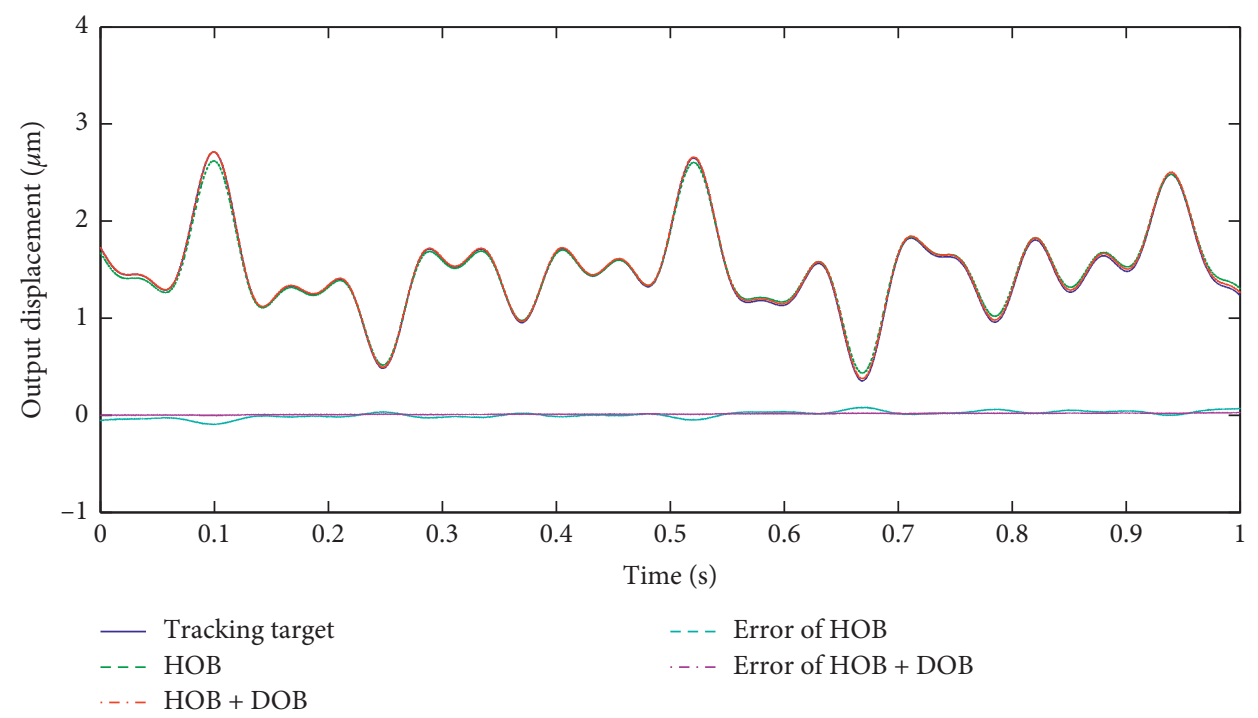

Figure 10: Control results in tracking a nonperiodical target.

absolute control error for the $\mathrm{HOB}$ and $\mathrm{HOB}+\mathrm{DOB}$ controllers are $0.259 \mu \mathrm{m}$ and $0.059 \mu \mathrm{m}$, respectively, which indicates the proposed $\mathrm{HOB}+\mathrm{DOB}$ control method can greatly improve the control system's antiinterference ability.
Anyway, from the experimental results, the HOB-based control can have a good capability for compensate the hysteresis with varying frequencies and amplitudes in a wide range. The DOB-based control method can restrain 


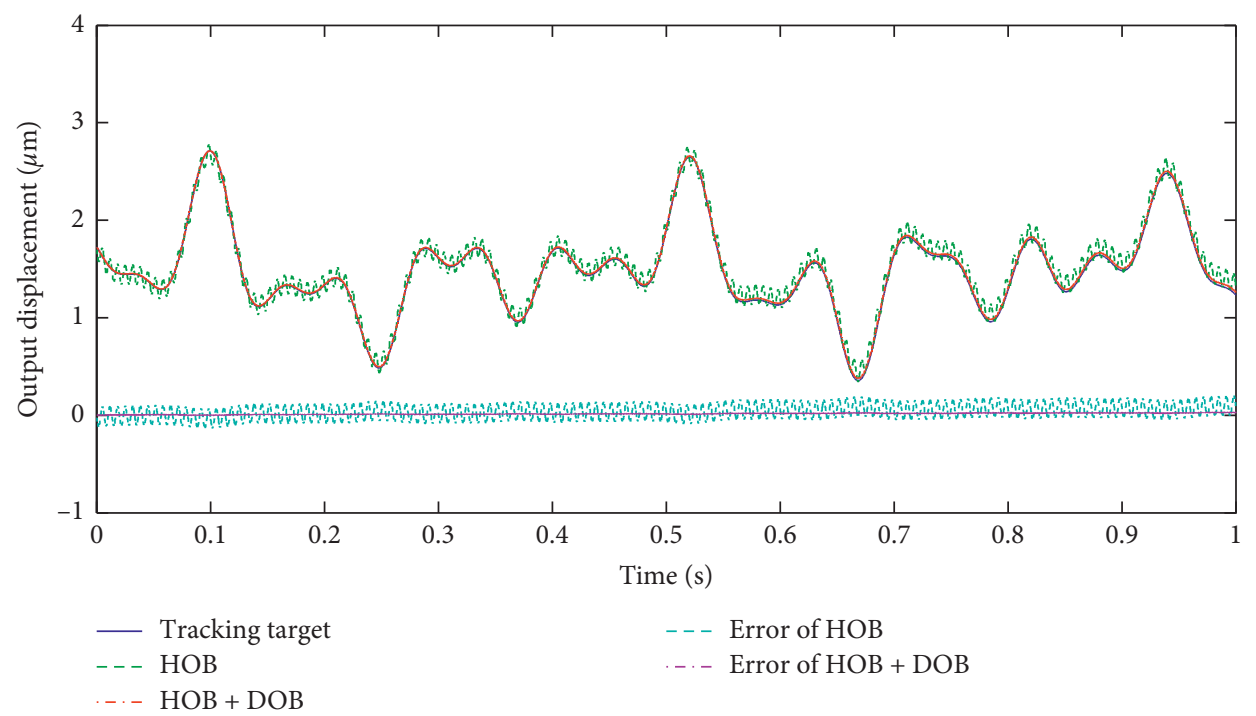

Figure 11: Control results in tracking a nonperiodical target with a sinusoidal disturbance.

disturbances, high-order unmodeled dynamics, and hysteresis compensation error.

\section{Conclusion}

We demonstrate a hysteresis and disturbances compensation method for a galfenol-actuated nanopositioning stage. In order to accurately describe the rate-dependent hysteresis, the proposed dynamic model considers the dynamic behavior of the power amplifier. Simplicity in implementation and robustness of performance are the main advantages of the proposed hysteresis and disturbances compensation approach. The effectiveness and efficiency of the method have been experimentally verified. The experimental results confirm that the proposed HOB-DOB-based compensation scheme successfully suppressed the hysteresis and disturbances of the galfenol-actuated nanopositioning stage to a satisfactory level under an input frequencies up to $100 \mathrm{~Hz}$.

\section{Data Availability}

The data used to support the findings of this study are available from the corresponding author upon request.

\section{Conflicts of Interest}

The authors guarantee that this work has no conflicts of interest to other research studies.

\section{Acknowledgments}

The authors wish to acknowledge the financial support by the Natural Science Foundation of Jiangsu Province (Grant no. BK20181301), National Natural Science Foundation of China (Grant no. 51975298), and Start-up Funding of Jiangsu University (Grant no. 18JDG026).

\section{References}

[1] P. G. Evans and M. J. Dapino, "State-space constitutive model for magnetization and magnetostriction of galfenol alloys," IEEE Transactions on Magnetics, vol. 44, no. 7, pp. 1711-1720, 2008.

[2] J. Atulasimha and A. B. Flatau, "A review of magnetostrictive iron-gallium alloys," Smart Materials and Structures, vol. 20, no. 4, Article ID 043001, 2011.

[3] M. R. Karafi, Y. Hojjat, and F. Sassani, "A new hybrid longitudinal-torsional magnetostrictive ultrasonic transducer," Smart Materials and Structures, vol. 22, no. 6, Article ID 065013, 2013.

[4] S. Karunanidhi and M. Singaperumal, "Design, analysis and simulation of magnetostrictive actuator and its application to high dynamic servo valve," Sensors and Actuators A: Physical, vol. 157, no. 2, pp. 185-197, 2010.

[5] L. Li, C. Zhang, B. Yan, L. Zhang, and X. Li, "Research of fastresponse giant magnetostrictive actuator for space propulsion system," IEEE Transactions on Plasma Science, vol. 39, no. 2, pp. 744-748, 2011.

[6] I. Chopra, "Status of application of smart structures technology to rotorcraft systems," Journal of the American Helicopter Society, vol. 45, no. 4, pp. 228-252, 2000.

[7] H. Hardee and N. L. Hardee, "Magnetostrictive-driven fretting machines for interconnection research," Journal of Alloys and Compounds, vol. 258, pp. 83-86, 1997.

[8] W. Zhu, L. Bian, G. Chen, S. Liu, Q. Zhou, and X. Rui, "Hysteresis modeling and experimental verification of a Fe-Ga alloy magnetostrictive actuator," Smart Materials and Structures, vol. 26, no. 8, Article ID 035039, 2017.

[9] F. Braghin, S. Cinquemani, and F. Resta, "A model of magnetostrictive actuators for active vibration control," Sensors and Actuators A: Physical, vol. 165, no. 2, pp. 342-350, 2011.

[10] W. Zhu, L. X. Bian, L. Cheng, and X. T. Rui, "Non-linear compensation and displacement control of the bias-rate-dependent hysteresis of a magnetostrictive actuator," Precision Engineering, vol. 50, pp. 107-113, 2017.

[11] X. Tan and J. S. Baras, "Modeling and control of hysteresis in magnetostrictive actuators," Automatica, vol. 40, no. 9, pp. 1469-1480, 2004. 
[12] G. Song, J. Zhao, X. Zhou, and J. DeAbreu-Garcia, "Tracking control of a piezoceramic actuator with hysteresis compensation using inverse Preisach model," IEEE/ASME Transactions on Mechatronics, vol. 10, no. 2, pp. 198-209, 2005.

[13] M. Al Janaideh, C.-Y. Su, and S. Rakheja, "Development of the rate-dependent Prandtl-Ishlinskii model for smart actuators," Smart Materials and Structures, vol. 17, no. 3, Article ID 035026, 2008.

[14] W. Zhu, G. L. Chen, L. X. Bian, and X. T. Rui, "Transfer matrix method for multibody systems for piezoelectric stack actuators," Smart Materials and Structures, vol. 23, no. 9, Article ID 095043, 2014.

[15] W. Zhu, L. X. Bian, Y. An, G. L. Chen, and X. T. Rui, "Modeling and control of a two-axis fast steering mirror with piezoelectric stack actuators for laser beam tracking," Smart Materials and Structures, vol. 24, no. 7, Article ID 075014, 2015.

[16] J. Yi, S. Chang, and Y. Shen, "Disturbance-observer-based hysteresis compensation for piezoelectric actuators," IEEE/ ASME Transactions on Mechatronics, vol. 14, no. 4, pp. 456464, 2009.

[17] S. M. Shahruz, "Performance enhancement of a class of nonlinear systems by disturbance observers," IEEE/ASME Transactions on Mechatronics, vol. 5, no. 3, pp. 319-323, 2000.

[18] W. Zhu and X.-T. Rui, "Hysteresis modeling and displacement control of piezoelectric actuators with the frequencydependent behavior using a generalized Bouc-Wen model," Precision Engineering, vol. 43, pp. 299-307, 2016. 


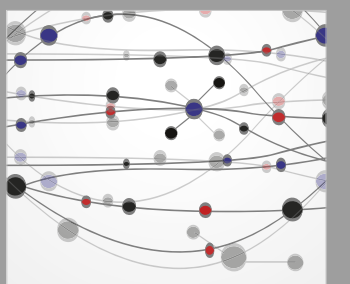

The Scientific World Journal
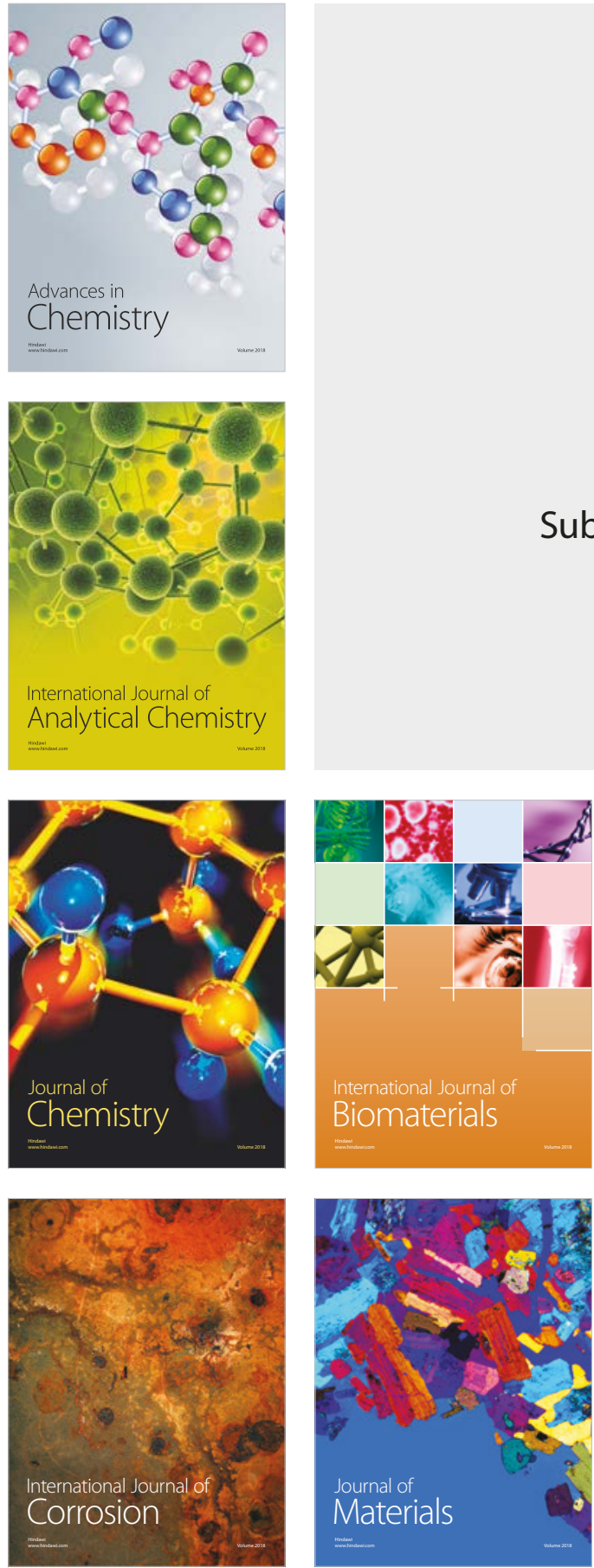

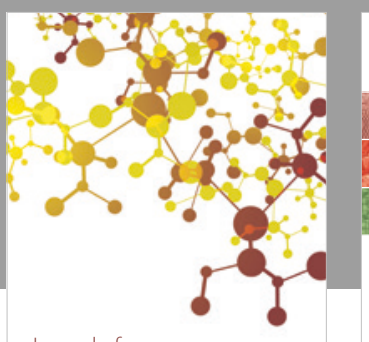

Journal of

Applied Chemistry
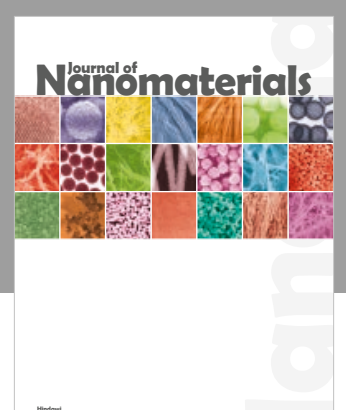

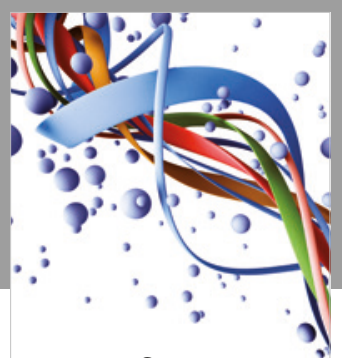

Scientifica

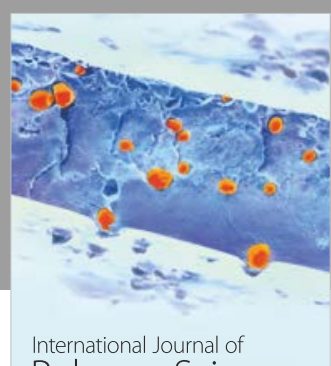

Polymer Science

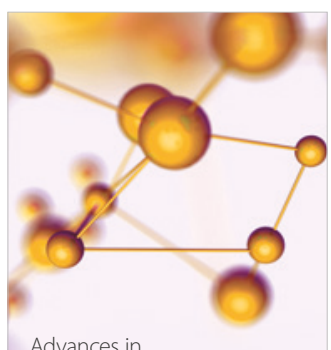

Physical Chemistry
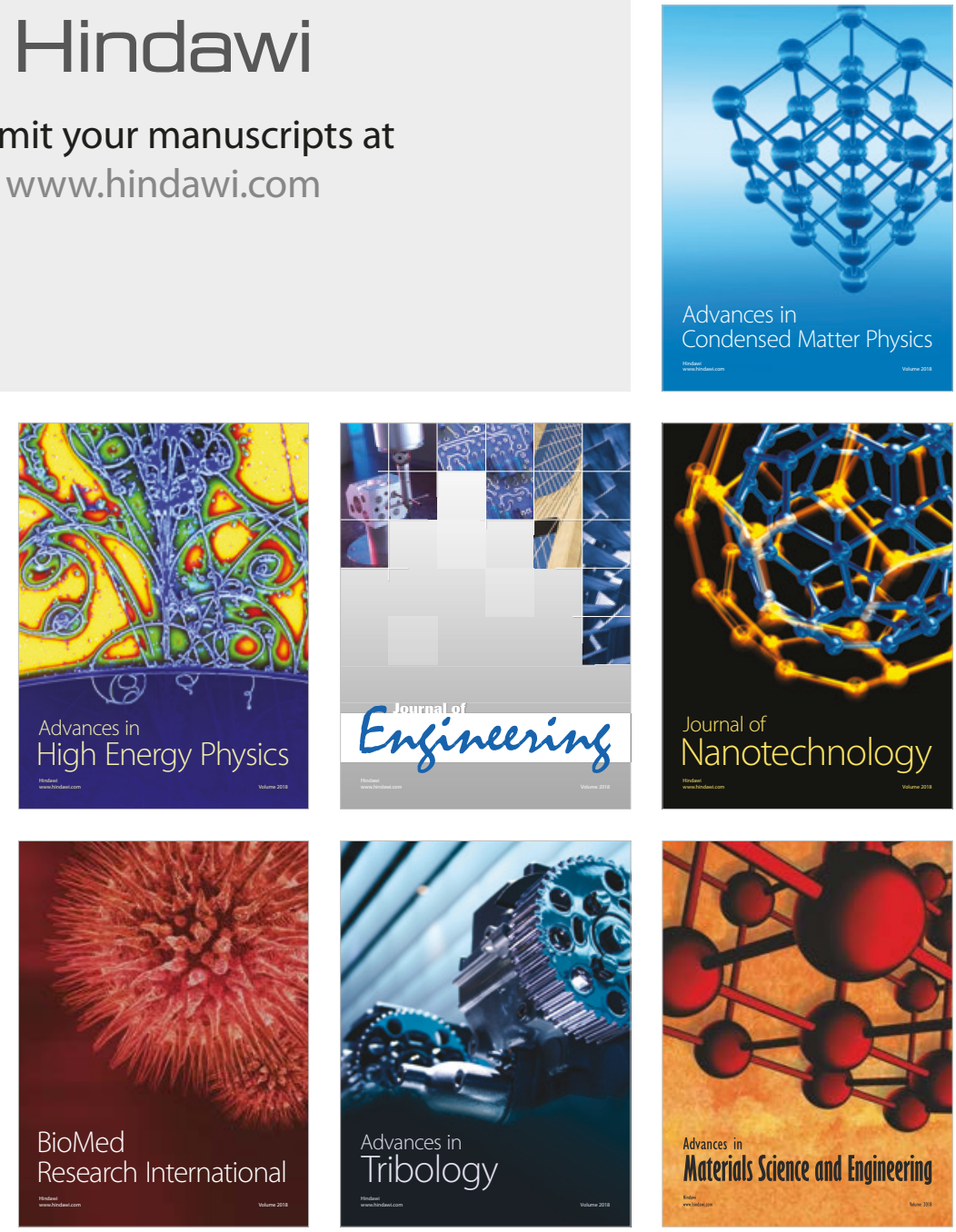